\title{
Editorial
}

\section{Therapeutic Options in Infections by Multidrug Resistant (MDR) Organisms}

Emergence of multidrug resistant (MDR) strains among common clinical microbes is now a great cause of concern throughout the world, including Bangladesh and its neighboring countries. ${ }^{1-6}$ It frequently causes treatment failure, prolonged period of hospital stay along with increased morbidity and mortality. ${ }^{7-9}$ History of development of drug resistance is quite colourful. In early forties of the last century, penicillin came to the limelight and industries responded to public demand by producing penicillin soaps, penicillin mouthwashes, penicillin lozenges, penicillin throat sprays and what not. Alexander Fleming, the discoverer of penicillin cautioned: "the greatest possibility of evil in selfmedication is the use of too small doses so that instead of clearing up infection, the microbes are educated to resist penicillin". ${ }^{10}$ Unfortunately, Fleming's words proved correct and within a few decades the world is shouldering a huge burden of emerging and re-emerging infectious diseases caused by multidrug resistant organisms.

Among the MDR organisms, MRSA (Methicillin resistant Staphylococcus aureus), VRE (vancomycin resistant Enterococci), ESBL (extended spectrum beta lactamase)producing Gram negative bacteria, Pseudomonas, Acinetobacter and Enterobacter species warrants special attention because of their limited therapeutic options. Until recently, only vancomycin provided effective therapy for MRSA infections. ${ }^{11}$ During nineties, there was virtually no antibiotic to treat infections caused by VRE. Similarly, therapeutic options are also limited for ESBL-producing Gram-negative bacilli, Acinetobacters and Stenotrophomonas species. $8,9,12$

When vancomycin is contraindicated or resistant, treatment of severe infections caused by MRSA can be done by teicoplanin, linezolid, daptomycin or tigecycline. In less severe cases, oral agents such as linezolid, rifampicin + fusidic acid, rifampicin + fluoroquinolone, co-trimoxazole, clindamycin and minocycline can be used.

Enterococcus, a normal flora of lower GIT and genital tract, frequently causes UTI, septicemia and intra-abdominal infections. Enterococci are intrinsically resistant to many antibiotics including cephalosporins, aminoglycosides, clindamycin and sometimes to tetracycline and erythromycin. Emergence of VRE has further complicated the treatment of Enterococcal infections. Quinupristin-dalfopristin, linezolid, daptomycin and tigecycline are the recent drugs used in systemic infections caused by VRE.13-14

Acinetobacter baumannii, present in the floor, water and environment of the hospitals, can cause pneumonia, bacteremia, endocarditis, meningitis and soft tissue infections among immunocompromized persons. They are mostly resistant to common antibiotics. Infections caused by MDR Acinetobacter are usually managed by imipenem or meropenem. Unfortunately, imipenem-resistant Acinetobacter has been reported throughout the world. Treatment of meropenem resistant Acinetobacter is difficult. Colistin and Polymyxin B are the only options. ${ }^{15}$

Pseudomonas aeruginosa found in soil, water and vegetations and sometimes in respiratory equipments, sinks and water taps, even in cleaning solutions, is an important nosocomial pathogen, specially when patient is immunocompromized and bed-ridden for long period.

Antipseudomonal drugs include piperacillin, mezlocillin and ticarcillin, ceftazidime, cefepime, aztreonam, imipenem, meropenem, tobramycin, gentamicin, amikacin, ciprofloxacin and levofloxacin. Unfortunately, due to development of resistance, the choice of drugs has been narrowed down. ${ }^{16}$

The ESBL producers are those Gram negative bacteria which produce enzymes that hydrolyze cephalosporines and aztreonam. Presently, over 150 such enzymes have been identified. Imipenem/ meropenem alone or in combination with aminoglycoside are the drug of choice for severe infections by ESBL-producing bacteria. ${ }^{12}$ However, cefepime, a fourth generation cephalosporin, may be useful in some of the cases. 
It is now obvious that the treatment for MDR organisms are becoming expensive and difficult. Antibiotics are to be prescribed only when needed. It is also important to pin-point the specific antibiotic which would suit patient the best. Time has come that health authorities should recognize the importance of establishing standard Microbiology laboratories for detection of MDR organisms in a hospital and in the community. But mere detection of the organisms would not be enough. Successful control of MDR needs improvements in hand hygiene, isolation of patients, enhanced environmental cleaning, active surveillance and education. It is hightime that our health authority would introduce a special module of infection control in the medical curriculum and make it a standard mandatory practice in all healthcare facilities.

\section{Zahidul Hasan}

Consultant, Microbiology, and Head, Infection Control Square Hospital, 18/F West Panthapath, Dhaka Mobile: 01819197339,E mail: zahidul@squarehospital.com

\section{References}

1. Basunia MRA, Rahman MR, Faruq MO, et al. Microbial pathogens and antibiotic sensitivity at intensive care unit of BIRDEM: a retrospective study. Bangladesh J Med 2005; 16 : 14-20.

2. Majumder D, Bordoloi JS, Phukan AC, Mahanta J. Antimicrobial susceptibility pattern among methicillin resistant staphylococcus isolates in Assam. Indian J Med Microbiol 2001; 19: 138-140.

3. Butt T, Ahmad RN, Usman M, Mahmood A. Methicillin-resistant Staphylococcus aureus, Pakistan, 1996-2003. [Letter] Emerg Infect Dis 2004; 10 (9): 1691-1692.

4. Corea E, de Silve T, Perera J. Methicillin-resistant Staphylococcus aureus: prevalence, incidence and risk factors associated with colonization in Sri Lanka. J Hosp Infect 2003; 55(2): 145-148.

5. McGowan JE Jr, Tenover FC. Control of antimicrobial resistance in the healthcare system. Infect Dis Clin North Am 1997; 11: $297-$ 311.

6. Schwartz B, Bell D, Hughes JM. Preventing the emergence of antimicrobial resistance: a call for action by clinicians, public health officials and patients. JAMA 1997; 278: 944-945.

7. Wilcox M. Healthcare-associated infection: morbidity, mortality and costs. Hosp Med 2004; 65(2): 88-91.

8. Stephen JW, Cynthia JK, Kari MG, Joyce EG, Helen MV, Rajiv S. Direct costs of multidrug-resistant Acinetobacter baumannii in the burn unit of a public teaching hospital. Am J Infect Control 2004; 32 (6): 342-344.

9. Ladman D, Quale JM, Myorga D, et al. Citywide Clonal Outbreak of Multiresistant Acinetobacter baumannii and Pseudomonas aeruginosa in Brooklyn, NY. Arch Intern Med 2002; 162: 1515-1520.

10. Levy SB. The Antibiotic Paradox, 2nd ed. Cambridge, Mass: Perseus Publishing; 2002.

11. Chang S, Sievert DM, Hageman JC, et al. Infection with Vancomycin-Resistant Staphylococcus aureus Containing the vanA Resistance Gene. N Engl J Med 2003; 348(14): 13421347.

12. Chaudhary U, Aggarwal R. Extended spectrum $\beta$-lactamases (ESBL) - An emerging threat to clinical therapeutics. Indian $\mathrm{J}$ Med Microbiol 2004; 22: 75-80.

13. Treitman AN, Yarnold PR, Warren J, Noskin GA. Emerging incidence of Enterococcus faecium among hospital isolates (1993 to 2002). J Clin Microbiol 2005; 43: 462-463.

14. Cetinkaya Y, Falk P, Mayhall CG. Vancomycin-resistant enterococci. Clin Microbiol Rev 2000; 13: 686-707.

15. Corbella X, Montero A, Pujol M, et al. Emergence and rapid spread of carbapenem resistance during a large and sustained hospital outbreak of multi-resistant Acinetobacter baumannii. J Clin Microbiol 2000; 38: 4086-4095.

16. Livermore DM. Multiple mechanisms of antimicrobial resistance in Pseudomonas aeruginosa: our worst nightmare. Clin Infect Dis 2002; 34: 634-640. 